\title{
Lo sviluppo di una realtà interculturale e plurilingue con il translanguaging italo-sloveno
}

\author{
Karin Ota \\ Scuola secondaria di primo grado con lingua d'insegnamento \\ slovena a Dolina, Italia \\ karin.ota@vspangerc.cloud
}

Le pratiche linguistiche moderne in realtà plurilingui e interculturali, come il contesto educativo italo-sloveno, possono contribuire in modo significativo a creare un ambiente di apprendimento inclusivo per tutti gli individui bilingui. Gli ambienti pedagogici sono dei contesti influenti che devono consentire l'espressione di tutti gli aspetti dell'identità di un individuo. È inoltre importante che delle adeguate pratiche linguistiche promuovano un'esperienza positiva della diversità personale e altrui e che questa particolarità venga considerata una fonte di arricchimento interpersonale. L'articolo analizza il ruolo degli attori pedagogici nel riconoscere e promuovere il plurilinguismo e l'interculturalità in un mondo super-diverso e sottolinea l'importanza di scegliere delle pratiche di insegnamento linguistico proficue e adeguate ai contesti educativi bilingui. Ci concentriamo soprattutto sulle potenzialità del translanguaging, un approccio didattico moderno già implementato in ambienti plurilingui stranieri, applicato al contesto scolastico dell'educazione bilingue italo-slovena.

Parole chiave: plurilinguismo, intercultura, translanguaging, bilinguismo, glottodidattica

(cc)BY-SA https://doi.org/10.26493/978-961-293-045-5.79-92

\section{Introduzione}

L'uomo è per natura un essere sociale e in base a tale sua caratteristica tende ad aggregarsi con altri individui e a costituirsi in una società. Con l'instaurazione di interconnessioni personali si dà origine a variegate comunità umane, che vengono in primo luogo definite tali in base ad un sistema di comunicazione comune, nel quale la lingua collettiva è il fattore principale di unione e appartenenza dei suoi vari membri. Se nell'epoca passata ad ogni Stato-nazione corrispondeva e veniva legittimata una comunità linguistica specifica e ben definita, nell'epoca moderna e contemporanea, con i grandi movimenti di emigrazione, immigrazione e con il fenomeno della globalizzazione, questa correlazione tra una determinata lingua e Stato 
non è di fatto ed eticamente più possibile. In ogni paese sono presenti individui, gruppi di persone o intere comunità che nella quotidianità alternano oppure sarebbero in grado di alternare la propria competenza comunicativa in più prime lingue. In tal caso si parla quindi di bilinguismo o plurilinguismo, fenomeni linguistici che caratterizzano storicamente gli Stati moderni nati nel XIX e nel Xx secolo, i quali si fondano al giorno d'oggi sulla Dichiarazione Universale dei diritti umani, sottoscritta dall'Assemblea Generale delle Nazioni Unite nel 1948. Accogliendo i principi di tale documento internazionale vennero sancite le libertà fondamentali di ogni essere umano, che deve avere la possibilità di costruire e coltivare liberamente ogni sfaccettatura della propria identità culturale, linguistica, religiosa o di qualsiasi altra condizione sin dalla nascita («Dichiarazione universale dei diritti umani» 1948). Dal punto di vista della lingua è stata a tal proposito sottoscritta nel 1996 la Dichiarazione universale dei diritti linguistici, che, tra i suoi vari articoli, indica che ogni persona appartenente a una determinata comunità o gruppo linguistico ha il diritto che le venga insegnata la propria lingua e cultura. Per le persone che fissano la propria residenza sul territorio di una comunità linguistica differente dalla loro, il documento menzionato esplicita che esse hanno il diritto e il dovere di avere un atteggiamento integrativo verso questa comunità, condividendo con essa delle sufficienti referenze, ma mantenendo allo stesso tempo le proprie caratteristiche culturali d'origine («Dichiarazione universale sui diritti linguistici» 2016). La diversità linguistica e culturale è uno dei tratti caratterizzanti dell'Unione europea e viene indicata in tutti i documenti sull'educazione come un valore da difendere e come una fonte di ricchezza (Coppola e Moretti 2018, 399).

Un punto di incontro di lingue e culture diverse è senza dubbio in prima istanza la scuola, che costituisce uno dei luoghi privilegiati per vivere la diversità linguistica e culturale come risorsa. Per la società europea attuale, la scuola e i contesti educativi del nuovo millennio devono svilupparsi e promuovere un ambiente inclusivo e interculturale, dove ogni individuo possa arricchire la propria identità con nuove conoscenze, esperienze e credenze legate anche a più culture e contesti sociali diversi. Nel caso di una persona bilingue o plurilingue la scelta o la necessità di usare una determinata lingua in una determinata circostanza non dovrebbe quindi essere condizionata da un contesto sottrattivo che favorisce un rapporto di dominazione e disequilibrio tra due culture e lingue diverse. Per rendere la diversità linguistica e culturale una reale risorsa e non una minaccia, occorrono adeguate pratiche di gestione delle differenze unite a programmi 
di educazione linguistica, nella sua accezione più ampia, e di formazione dell'interculturalità (Coppola e Moretti 2018). Il concetto di lingua e di cultura non deve essere pensato secondo una visione chiusa e monolitica di esse, ma piuttosto bisogna promuovere il contatto e il contagio linguistico e culturale con processi di scambio.

Le pratiche linguistiche, gli approcci e le metodologie didattiche utilizzate in contesti educativi e scolastici dovrebbero quindi essere volti a conservare, ampliare e interrelazionare i diversi bagagli culturali, linguistici e sociali di ogni singolo individuo e di diversi individui tra di loro, che siano essi monolingui o bilingui, monoculturali o multiculturali. Compito della scuola è quindi di coltivare, sviluppare e riconoscere il valore intrinseco dell'interculturalità e abbandonare la strada del multiculturalismo inteso come mosaico di culture separate l'una dall'altra e come concetto che promuove un approccio basato sulla mera tolleranza dell'altro (Coppola e Moretti 2018). In tali contesti l'insegnante può intervenire e modificare soprattutto le variabili ambientali, agendo sul contesto educativo, selezionando, modificando e utilizzando l'input nel modo pedagogicamente più adeguato, restando però ben consapevoli dell'interrelazione tra i vari fattori e le variabili che influenzano l'apprendimento (Diadori 2016, 31-32).

\section{L'apprendimento linguistico nella super-diversità}

Tra i fattori cruciali nell'apprendimento di una lingua possiamo citare le variabili linguistiche, ambientali e individuali che un soggetto possiede o a cui è esposto (Diadori 2016). Al giorno d'oggi, però, con le trasformazioni sociali innescate da cambiamenti della migrazione globale, molti individui sono immersi in un'ambiente di alta complessità sociale e tali realtà multiculturali determinano la necessità di porre maggiore attenzione ai temi dell'educazione linguistica e riorientare alcuni approcci linguistici fondamentali al fine di affrontare e comprendere meglio formazioni sociali complesse e non più necessariamente vincolate agli Stati-nazione come succedeva soprattutto nel XIX secolo. Vertovec (2017) propone il concetto di «super-diversità» che introduce per descrivere e analizzare il contesto di rapido cambiamento demografico londinese all'inizio del XXI secolo e gli effetti delle migrazioni che hanno portato ad un'alta complessità sociale. Nel mondo sempre più persone di varie culture e gruppi linguistici vengono in regolare contatto una con l'altra, soprattutto nelle città metropolitane. La super-diversità è il risultato della mobilità delle persone ovvero della mobilità delle risorse. Più che una descrizione della diversificazione della diversità come risultato delle recenti migrazioni, la super-diversità ha il 
potenziale di offrire una prospettiva interdisciplinare sul cambiamento e sulla complessità sociale instauratasi (Deumert 2014). Tale concetto fa capire la diversità non soltanto in termini di raggruppamento etnico, raziale o nazionale, ma ha anche lo scopo di sradicare la vecchia visione binaria che opponeva la prevalente cultura maggioritaria alla cultura minoritaria degli immigrati. La super-diversità come teoria della prassi vuole criticare le forze di discriminazione e far emergere delle specifiche configurazioni di diversi sfondi nazionali, raziali o etnici, di genere, età, lingua, status socio-economico, stato legale e canale migratorio ovvero le congiunzioni o interazioni tra queste diverse variabili degli individui. Ha quindi lo scopo di riconoscere la complessità della diversità della società. Ma piuttosto che offrire una descrizione delle differenze, la super-diversità rappresenta un orientamento ideologico alla diversità e ai processi di diversificazione. La prospettiva di tale teoria fa emergere un punto di partenza per descrivere il cambiamento della popolazione e per identificare l'interazione di differenti dimensioni della diversità (Ram et al. 2012). La super-diversità richiede però un approccio interdisciplinare, ovvero transdisciplinare, allo scopo di generare dei nuovi saperi in ambienti super-diversi. I vari ambiti disciplinari forniscono un quadro di come la ricerca che si focalizza sulla super-diversità sia in grado di fare luce sui processi sociali, nei quali la lingua è il fattore sul quale viene posta la maggiore attenzione. Unendo la super-diversità alla lingua la ricerca di fondo si basa sulla problematica di come le persone comunicano nelle società caratterizzate da un'intensa diversità e complessità sociale al fine di sviluppare delle nuove forme di comunicazione in quanto le trasformazioni sociali vanno a braccetto con le trasformazioni socio-linguistiche. In tale prospettiva si parla dunque della super-diversità socio-linguistica che nasce dal contatto tra nazionalità, etnie, lingue, modelli di cultura e pratiche diverse, interazioni che sono ormai una norma nei paesi sviluppati (De Fina, Ikizoglu e Wegner 2017).

La super-diversità si esprime in vari contesti sociali, ma negli Stati moderni essa è particolarmente visibile negli ambienti scolastici di ogni ordine e grado. Negli ultimi anni gli insegnanti devono fronteggiare sempre più spesso delle classi di discenti che hanno già avuto esperienza di studio o di acquisizione anche di altre lingue straniere e si ritrovano quindi ad operare in classi multilingui (Mezzadri 2015, 124). In classi del genere si sviluppano molto spesso dei modelli d'azione che portano all'introduzione di un'altra lingua come lingua della comunicazione, mentre la lingua studiata assume un ruolo secondario ed è usata in modo artificioso solo attraverso le attività create per il contesto didattico. Mezzadri $(2015,127)$ infatti propo- 
ne una riflessione sul fatto che «molto spesso l'insegnamento linguistico è schiacciato sull'acquisizione di abilità comunicative interpersonali di base e fatica a espandersi verso un uso che investa ambiti culturali anche diversi». La lingua studiata deve essere in primo luogo usata come strumento di comunicazione in classe, anche se è necessario tenere in mente che imporre un uso esclusivo della lingua seconda può influire negativamente sull'apprendimento di individui che hanno difficoltà a capire e a esprimersi nella lingua studiata, il che porterebbe all'insorgere di filtri affettivi, oggetto di studio di Krashen (1981). Anche dal punto di vista dei contenuti studiati, in caso gli studenti non posseggano degli strumenti linguistici sufficienti per affrontarli, si rischia in tale situazione di non riuscire ad approfondire tali tematiche; in questo caso è necessario esplicitare determinati concetti nella lingua più vicina agli studenti. Lo stesso vale per riassumere o spiegare delle nozioni grammaticali che, spiegate solo in L 2, non verrebbero capite appieno da tutti gli studenti. Per comunicare significati rapidamente ed efficacemente l'insegnante può avvalersi anche della traduzione, stando attendo a non abusarne per non incorrere in comprensioni globali sbagliate che non prenderebbero in considerazione il contesto, la funzione di una determinata parola o l'interpretazione di essa in base ad altri parametri (Mezzadri 2015, 128).

Questi sviluppi hanno influenzato i modi in cui le persone comunicano in tutti i contesti della loro vita e quindi portano al rimodellamento di concetti e metodologie. Un esempio glottodidattico può essere rappresentato dalla pratica linguistica dell'eteroglossia e del translanguaging (Blackledge e Creese 2017, 1-14). La prima presuppone di identificare come i soggetti facciano un uso simultaneo di diversi tipi di discorso o linguaggio che rispecchiano specifici modi di essere e di vedere il mondo (Blackledge e Creese 2014), il secondo invece è un approccio all'insegnamento che tiene conto della complessità linguistica in cui viviamo e assume che la generazione di significato, soprattutto delle persone bi- o plurilingui, avvenga utilizzando tutti i repertori linguistici a loro disposizione (Otheguy, Garcia e Reid 2015, 281).

\section{Il translanguaging come pratica glottodidattica di promozione dell'interculturalità e del plurilinguismo}

La diversità in tutte le sue declinazioni, che costituisce ormai una caratteristica strutturale delle istituzioni educative, può diventare un terreno fertile per la sperimentazione di modalità didattiche cooperative che promuovano il plurilinguismo e il confronto interculturale. In tali contesti si 
deve rispondere alle difficoltà metodologico-didattiche (che si riscontrano nelle scuole come conseguenza di contesti didattici marcatamente eterogenei dal punto di vista linguistico-culturale) accogliendo le potenzialità di alcune tecniche dell'approccio dialogico, che favoriscono atteggiamenti positivi nei confronti delle differenti lingue e culture. Una delle pratiche linguistiche che si sta sviluppando e sta seguendo un sentiero ancora relativamente inesplorato all'interno dell'educazione bilingue e interculturale è il translanguaging, che viene proposto da Garcia nel 2009 come approccio all'insegnamento in generale e nella glottodidattica e viene fatto proprio da diversi studiosi di pedagogia bi- e plurilingue (tra cui Creese e Blackledge 2010; 2011; Canagarajah 2011; Cenoz e Gorter 2015; Paulsrud et al. 2017). Tale pratica parte dal presupposto che i soggetti bilingui nel comunicare non utilizzano due sistemi linguistici distinti e autonomi, come è stato tradizionalmente assunto, ma hanno invece un unico repertorio linguistico che utilizzano trasversalmente in termini sia culturali che linguistici (Lewis, Jones e Baker 2012). Promovendo l'approccio glottodidattico del translanguaging bisogna quindi innanzitutto distinguere tra due termi$\mathrm{ni}$, il plurilinguismo e il multilinguismo. Soltanto il primo è collegato a un concetto di competenza linguistica ampio che non tiene le diverse lingue e culture separate in diversi compartimenti mentali, ma al contrario ingloba un insieme di conoscenze, abilità e strategie interrelate che il parlante plurilingue rievoca in situazioni diverse per garantire una comunicazione efficace con un determinato interlocutore. Il multilinguismo si riferisce invece alla coesistenza di più lingue, considerate però in modo distinto, separate l'una dall'altra cognitivamente e pragmaticamente (Coppola 2016). 11 translanguaging si basa quindi sul concetto di plurilinguismo e viene definito come «una modalità linguistica plurilingue socialmente determinata con la quale generiamo le nostre identità» (Capua 2015) e con esso ci riferiamo a nuove pratiche linguistiche che rendono nota la complessità degli scambi linguistici tra persone con storie e retroscena di vita diversi. Assumendo come valida tale pratica è indispensabile prediligere e promuovere una visione non-essenzialista della cultura (Holliday 2014) e della lingua, approccio che presuppone di dover capire la complessità di chiunque faccia parte di un gruppo culturale o linguistico con cui non abbiamo una pregressa familiarità, ma con cui vogliamo instaurare una comunicazione eticamente corretta ed efficace. Secondo questa visione le culture sono dei flussi che possono mescolarsi e interconnettersi tra di loro, essendo caratterizzate da confini sfocati e non restando costrette entro le barriere delle frontiere nazionali. Con il translanguaging si rende possibile e più comple- 
ta la comprensione di significati translinguistici che non assumerebbero la stessa valenza se generati prediligendo negli individui bilingui un'unica identità linguistica e culturale fissa e vincolata dagli Stati nazionali (Garcia e Wei 2014, 21). Perché ciò avvenga sarebbe necessario accettare un approccio olistico nei contesti educativi e didattici, dove però, al giorno d'oggi, la lingua accademica è ancora concepita esclusivamente come monolingue anche nei paesi dichiarati costituzionalmente come bilingui (Capua 2015; Cenoz e Gorter 2017, 901). Anche nelle scuole bilingui o con lingua d'insegnamento diversa dalla lingua della nazione, le lezioni vengono impartite o in una o nell'altra lingua, tenendo le due lingue separate e non approvando un insegnamento linguisticamente misto, con il quale si userebbe in modo consapevole il mescolamento o l'alternanza delle due lingue nella stessa lezione o momento educativo. I contesti didattici che prevedono il translanguaging invece promuovono non solo l'accettazione, ma anche lo sviluppo di una realtà plurilingue. Secondo questa pratica, però, le diverse lingue non vengono tenute separate, come avviene di solito nei contesti bilingui, dove si impone di usare una determinata lingua in base a determinati criteri di scelta (per esempio l'argomento di studio, una materia, un insegnante, una specifica lezione di lingua ecc.), ma viene al contrario promosso uno sviluppo completo e simultaneo dell'intero repertorio linguistico della persona bilingue, senza porre dei confini tra le due lingue. L'uso pedagogico del termine si riferisce quindi all'uso pianificato e sistematico di due lingue all'interno di un'unica lezione, per esempio specificando e variando le lingue nei momenti di produzione o ricezione linguistica (per esempio l'ascolto e la lettura avvengono in una lingua, la produzione orale e scritta in un'altra lingua). Questo tipo di pratica riflette l'idea che i bambini bilingui fanno un uso pragmatico di entrambe le lingue per massimizzare la comprensione e implementare la loro abilità comunicativa e, tenendo conto di tale assunto, il translanguaging si basa proprio sulle pratiche linguistiche e conversazionali implicite ed esplicite che usano i bilingui. I contesti didattici in cui viene usato il translanguaging promuovono l'applicazione di tali pratiche nell'insegnamento e nell'educazione al fine di eliminare le barriere linguistiche e culturali che possono portare all'isolamento, all'esclusione e all'ostacolamento dell'apprendimento di un individuo che non ha piena padronanza di una determinata lingua di insegnamento o che proviene da un contesto culturale diverso (Garcia e Wei 2014, 97).

Come precedentemente menzionato, il bilinguismo o plurilinguismo è un fenomeno che si sviluppa in ambienti storicamente multiculturali o come conseguenza di processi migratori, ma esso nasce anche nelle zone 
etnicamente miste e dal rapporto di vicinato o di confine tra due nazioni di lingua ufficiale diversa. Tale bilinguismo o plurilinguismo è di solito rappresentato dalle minoranze linguistiche e nazionali, che, per questioni storiche, politiche o sociali, sviluppano e promuovono l'uso della propria lingua d'origine al di fuori dei confini dello Stato dove tale lingua assume la funzione di lingua ufficiale della nazione. Per una minoranza linguistica o nazionale è di fondamentale importanza il diritto di imparare la madrelingua e il diritto di fruirne per l'educazione, perché solo così si può conservare la lingua stessa e trasmettere il patrimonio culturale alle generazioni future (Pertot 2018).

\section{Il translanguaging nei contesti educativi italo-sloveni}

Un esempio di comunità bilingue di questo genere nasce dai territori del confine italo-sloveno ed è rappresentato dalla minoranza nazionale slovena nella regione Friuli Venezia Giulia in Italia e dalla minoranza nazionale italiana nella zona costiera del Litorale in Slovenia. In qualsiasi Stato dove vivono delle minoranze la lingua minoritaria può essere introdotta nel sistema scolastico in forme diverse: come materia supplementare, facoltativa, obbligatoria, come lingua straniera, oppure come lingua d'insegnamento veicolare o per un numero limitato di lezioni (Pertot 2018). Nel caso specifico delle citate due minoranze slovene e italiane, la lingua minoritaria è la lingua d'insegnamento della scuola e la lingua maggioritaria viene invece studiata come materia obbligatoria in modo simile alla lingua straniera (determinate ore alla settimana). L'introduzione nel sistema scolastico della lingua minoritaria nella forma di lingua d'insegnamento può essere vista come scelta storica per garantire una maggiore valorizzazione della lingua e della cultura del gruppo di minoranza. Nel passato vari conflitti e polemiche burrascose di carattere storico-politico hanno ostacolato una convivenza di confine feconda e pacifica tra le due comunità, questo perché le due minoranze nazionali citate, anche se ufficialmente venivano riconosciute e tutelate da apposite leggi nazionali, si videro spesso costrette a dover legittimare in vari modi la propria presenza sul territorio. L'uso di un'unica lingua d'insegnamento nelle scuole delle minoranze è quindi storicamente legato anche alla costante sensazione di un'incombente minaccia e pericolo di omologazione e dominazione da parte della lingua e della cultura maggioritaria. Se queste scuole erano in passato concepite come luoghi dedicati prevalentemente all'educazione degli alunni appartenenti alla determinata minoranza, al giorno d'oggi le lingue di minoranza in cui avviene la scolarizzazione possono rappresentare per gli alunni la prima 
lingua, la seconda lingua o anche una lingua del tutto straniera (Mikolič, Pertot e Zudič Antonič 2006).

In base ai cambiamenti menzionati, gli insegnanti di tali scuole si ritrovano spesso a operare in classi linguisticamente non omogenee, dove lo sloveno e l'italiano si alternano il ruolo di prima lingua, seconda lingua o lingua straniera. In circostanze del genere, per garantire una didattica efficace e la comprensione dei contenuti da parte di tutti gli individui, gli insegnanti si ritrovano a dover adattare i propri metodi e le proprie pratiche di insegnamento. A tal proposito succede per esempio che utilizzino dei materiali didattici specifici o si avvalgono della traduzione e della spiegazione semplificata dei contenuti per farsi capire meglio e per rinforzare l'apprendimento da parte di tutti gli alunni. Anche se queste modalità d'insegnamento non trovano posto e non sono ufficialmente approvate nelle linee guida del curricolo scolastico in quanto, come d'altronde anche presso altre minoranze, si crede erroneamente (Cenoz e Gorter 2017) che possano concorrere all'erosione della lingua minoritaria, gli insegnanti che hanno come alunni dei bambini bilingui tendono comunque (anche inconsapevolmente) a utilizzare in classe delle tecniche e metodi didattici personalizzati e individualizzati a seconda del tipo e grado di varietà linguistica o culturale della classe in cui insegnano. Una pratica che viene non dichiaratamente usata nelle scuole menzionate è anche il translanguaging, che permette, nel caso specifico delle scuole in questione, di costruire il significato o una migliore comprensione dei contenuti studiati sia nella lingua slovena che nella lingua italiana. Tale pratica presuppone l'esistenza di un unico repertorio linguistico nei soggetti bilingui, che essi utilizzano trasversalmente in modo inconsapevole. Cummins (1979) ha elaborato la teoria dell'interdipendenza linguistica nella quale espone la sua visione che le pratiche linguistiche o metalinguistiche di una lingua possano essere trasferite ad un'altra lingua (Vogel e Garcia 2017). Il bilinguismo è quindi caratterizzato dalla dinamicità e il repertorio linguistico viene interiorizzato tramite delle interazioni sociali nelle quali il soggetto seleziona translinguisticamente gli elementi linguistici di qualsiasi lingua per produrre un pieno significato. Anche se gli insegnanti non si sentono legittimati ad ammodernare gli approcci didattici in questa direzione per il timore di prediligere una lingua a discapito dell'altra, è inevitabile che tali pratiche linguistiche vengano adoperate per garantire un ambiente inclusivo e il più alto grado di comprensione e conseguente apprendimento per tutti gli alunni. La scuola deve infatti basarsi su due principi basilari: l'integrazione culturale e l'inclusione (Coppola e Moretti 2018, 399). Il primo principio è la condizione perché 
gli alunni possano partecipare in modo paritario e collaborativo al comune processo di costruzione delle conoscenze e delle competenze e ciò non può avvenire se l'alunno non ha la possibilità di fare uso del suo intero repertorio linguistico e delle abilità già acquisite, anche se in un'altra lingua. Il secondo principio rimanda a un'accezione ampia di inclusione, e quindi anche di diversità, che tiene conto del diritto a un percorso personalizzato, che differisce in base alle diverse caratteristiche culturali e linguistiche dell'individuo. Per rendere operativo l'obiettivo di una formazione alla diversità occorrono degli spazi di apprendimento cooperativi e plurilingui, all'interno dei quali possa realizzarsi un dialogo costruttivo tra diversi (per lingua, cultura, bisogni, abilità) che costituisce uno dei principali obiettivi della competenza interculturale (Byram 2019), intesa come capacità di comunicare (e quindi non solo di esprimersi) in modo efficace e appropriato con altre persone di altre culture (Coppola 2016). Il translanguaging è quindi una pratica che favorisce l'idea della linguacultura, concetto che assume che una lingua non include soltanto gli elementi come la grammatica e il lessico, ma anche le conoscenze, le informazioni culturali e le abitudini personali. Ogni qualvolta un individuo esplora un contesto al di fuori della sua lingua madre, per esempio esprimendosi in una seconda lingua o avendo con essa un contatto autentico, esso si ritrova a creare una nuova complessità culturale e a dar vita ad un'identità multiforme, con la quale accetta una continua rinegoziazione e ricostruzione del proprio essere (Holliday 2014).

\section{Conclusioni}

Gli approcci linguistici volti a valorizzare l'interculturalità, tra i quali abbiamo presentato il translanguaging, risultano quindi essere la chiave che consente all'individuo plurilingue una piena espressione della propria identità. Con la pratica del translanguaging nelle scuole slovene in Italia e nelle scuole italiane in Slovenia si potrebbe dunque aspirare alla legittimazione della creazione di un ambiente interculturale e plurilinguistico privo di stereotipi e pregiudizi etnici, che sono purtroppo ancora presenti tra i membri della comunità nazionale slovena in Italia e quella italiana in Slovenia. Si potrebbe così dimostrare come gli elementi delle due culture sono intrecciati in modo molto forte ed esercitare un influsso positivo sulla formazione dell'identità culturale dell'individuo (Mikolič, Pertot e Zudič Antonič 2006, 41), senza che esso si senta obbligato a dover scegliere o prediligere una sfaccettatura della propria identità legata solo ad una cultura e lingua. L'approccio del translanguaging nel contesto italo-sloveno potrebbe 
accrescere le competenze e conoscenze linguistiche di tutti i soggetti coinvolti, creare una reale comunicazione e rendere visibili le relazioni tra di esse, portando alla promozione di un dialogo positivo e di una interazione sociale tra questi due mondi e culture diverse. Riducendo le distanze tra di esse e ricollocando le dinamiche nell'interazione si permetterebbe di creare un autentico e reciproco dialogo interculturale e plurilinguistico, dove entrambe le lingue e culture sarebbero dei co-attori nella modellazione dell'identità multiforme dei soggetti bilingui italo-sloveni.

\section{Riferimenti bibliografici}

Blackledge, A., e A. Creese. 2014. «Heteroglossia as a Practice in Pedagogy». In Heteroglossia as a Practice and Pedagogy, a cura di A. Blackledge e A. Creese, 1-20. Londra: Springer.

- 2017. Language and Superdiversity: An Interdisciplinary Perspective. New York: Routledge.

Byram, M. 2019. «Intercultural Language Teaching in an Era of Internationalization.» In Intercultural Foreign Language Teaching and Learning in Higher Education Contexts, a cura di P. Romanowsky e E. Bandura, 99-120. Hershey, PA: IG I Global.

Canagarajah, S. 2011. «Translanguaging in the Classroom: Emerging Issues for Research and Pedagogy.» Applied Linguistics Review 2:1-28.

Capua, C. 2015. «O. García, L. Wei, Translanguaging: Language, Bilingualism and Education». Bollettino Itals, n. 6o. https://www.itals.it/recensione/ o-garc\% $\mathrm{C}_{3} \% \mathrm{ADa}$-l-wei-translanguaging-language-bilingualism -and-education

Cenoz, J., e D. Gorter, a cura di. 2015. Multilingual Education: Between Language Learning and Translanguaging. Cambridge: Cambridge University Press.

- 2017. «Minority Languages and Sustainable Translanguaging: Threat or Opportunity?» Journal of Multilingual and Multicultural Development 38 (10): 901-912.

Coppola, D. 2016. «Parlo cinese, sono toscano, studio inglese: il diritto al plurilinguismo nelle scuole multietniche.» Scienza e pace 7 (3): 1-16.

Coppola, D., e R. Moretti. 2018. «Valorizzare la diversità linguistica e culturale: uno studio di caso.» Studi e ricerche 13:397-412.

Creese, A., e A. Blackledge. 2010. «Translanguaging in the Bilingual Classroom: A Pedagogy for Learning and Teaching?» The Modern Language Journal 94 (1): 103-115.

- 2011. «Separate and Flexible Bilingualism in Complementary Schools: Multiple Language Practices in Interrelationship.» Journal of Pragmatics 43 (5): 1196-1208. 
Cummins, J. 1979. «Linguistic Interdipendence and the Educational Development of Bilingual Children.» Review of Educational Research 49 (2): 222-251.

De Fina, A., D. Ikizoglu e J. Wegner. 2017. Diversity and Super-Diversity Sociocultural Linguistic Perspectives. Washington, D c: Georgetown University Press.

Deumert, A. 2014. «Digital Superdiversity: A Commentary.» Discourse, Context and Media 4 (5): 116-120.

Diadori, P. 2016. «Le Variabili nell'Apprendimento della L 2.» In Insegnare italiano a stranieri, a cura di P. Diadori, 2-34. Milano: Mondadori.

«Dichiarazione universale dei diritti umani.» 1948. https://www.ohchr.org/ EN/UDHR/Documents/UDHR_Translations/itn.pdf.

«Dichiarazione universale sui diritti linguistici.» 2016. https://arlef.it/app/ uploads/documenti/Dichiarazione_Universale_sui_Diritti_Linguistici _1996_-IT-.pdf.

García, O. 2009. Bilingual Education in the 21st Century: A Global Perspective. Malden, MA: Wiley-Blackwel.

García, O., e L. Wei. 2014. Translanguaging: Language, Bilingualism and Education. New York: Palgrave MacMillan.

Holliday, A. 2014. «Cultural Travel and Cultural Prejudice.» In Identities: Representation and Practices, a cura di M. B. Aquino e S. Frota, 25-44. Lisbona: University of Coimbra.

Krashen, S. D. 1981. Second Language Acquisition and Second Language Learning. Oxford: Pergamon.

Lewis, G., B. Jones e C. Baker. 2012. «Translanguaging: Origins and Development from School to Street and Beyond.» Educational Research and Evaluation 18 (7): 641-654.

Mezzadri, M. 2015. «La gestione della classe.» In I nuovi ferri del mestiere, a cura di M. Mezzadri, 117-148. Torino: Loescher.

Mikolič, V., S. Pertot e N. Zudič Antonič. 2006. Med kulturami in jeziki/Tra lingue e culture. Capodistria: Annales.

Otheguy, R., O. Garcia e W. Reid. 2015. «Clarifying Translanguaging and Deconstructing Named Languages: A Perspective from Linguistics.» Applied Linguistics Review 6 (3): 281-307.

Paulsrud, B. A., J. Rosén, B. Straszer e Å. Wedin. 2017. New Perspectives in Translanguaging and Education. Bristol: Multilingual Matters.

Pertot, S. 2018. «Le scuole statali con lingua d'insegnamento slovena e i corsi di lingua slovena per adulti». Jezik-Lingua. http://www.jezik-lingua.eu/ it/14043/Chi-puo-frequentare-le-scuole-statali-con-lingua-d -insegnamento-slovena-nelle-province-di-Trieste-e-di-Gorizia-e-la -scuola-statale-con-insegnamento-bilingue-sloveno-italiano-di-San -Pietro-al-Natisone. 
Ram, M., K. Trehan, J. Rouse, T. Jones, e K. Woldesenbet. 2012. «Ethnic Minority Business Support in the West Midlands: Challenges and Developments.» Enviroment and Planning C: Government and Policy 30:504-519.

Vertovec, S. 2017. «Mooring, Migration Milieus and Complex Explanations.» Ethnic and Racial Studies 40 (9): 1574-1615.

Vogel, S., e O. García. 2017. "Translanguaging.» In Oxford Research Encyclopedia of Education, a cura di G. Noblit, 44-45. Oxford: Oxford University Press.

\section{Italijansko-slovenski translanguaging kot sredstvo razvoja medkulturnosti in večjezičnosti}

Avtorica se v članku osredotoča na dvojezično učenje jezikov in možnost uvajanja sodobnih jezikovnih praks, kot je t.i. translanguaging, v italijanskoslovenski obmejni izobraževalni kontekst v Italiji in Sloveniji. Jezikovna in kulturna raznolikost je $\mathrm{v}$ teh in drugih dvojezičnih okoljih ena od značilnosti današnje družbe in pomembno je, da jo obravnavamo kot vir bogastva in kot vrednoto, ki jo moramo ohranjati tudi v bodoče. Izobraževalni konteksti so privilegirani kraji, kjer se lahko to raznolikost doživlja pozitivno in kjer se mora posamezniku omogočati, da izraža vse vidike svoje jezikovne in kulturne identitete. S primernimi in individualiziranimi praksami mu je treba zagotoviti, da je del inkluzivnega okolja in da pozitivno goji svojo večkulturno identiteto. V večjezičnih kontekstih, kot je manjšinsko italijanskoslovensko okolje v Italiji in Sloveniji, na katerega smo se osredotočili v tem prispevku, se pogosto uporabljajo subtraktivne metode poučevanja ali prakse, ki od dvojezičnih učencev zahtevajo, da izmenjujejo izražanje jezikovnih in kulturnih prvin glede na določeno učno situacijo, predvsem glede na učni jezik. Sodobne jezikovne prakse, kot je translanguaging, omogočajo, da presežemo subtraktivno interpretacijo večjezičnega učenja, da si prizadevamo za legitimizacijo medkulturnosti in večjezičnosti ter za preseganje kakršnegakoli stereotipa ali predsodka, ki je povezano s tema dvema pojmoma. V članku smo nazadnje poudarili možnost učinkovite uporabe jezikovne prakse translanguaging-a v dvojezičnem italijansko-slovenskem kontekstu za ustvarjanje in zagotavljanje pozitivnega medkulturnega učnega okolja.

Ključne besede: večjezičnost, medkulturnost, translanguaging, dvojezičnost, glotodidaktika

\section{The Development of an Intercultural and Multilingual Reality with the Italian-Slovene Translanguaging}

In this article the author focuses on the bilingual language learning and on the possibility of introducing modern linguistic practices, such as the 
translanguaging, in the Italian-Slovenian educational context in border areas in Italy and Slovenia. Like in these two bilingual enviroments, linguistic and cultural diversity is one of the characteristic features of today's society and it must be seen as an enrichment and as a value to be defended. Educational contexts are among the privileged places to experience this diversity as a resource and where, by allowing learners to express every facet of their linguistic and cultural identity, they are guaranteed to be part of an inclusive enviroment that allows for the development of their interculturality to be positively promoted. In multilingual contexts, such as the Italian-Slovenian in Italy and Slovenia, that we focused on in the paper, subtractive teaching methods are often used as well as the kind of teaching practices that forces bilingual learners to alternate the expression of the linguistic and cultural contents according to the teaching context, particularly to the language of instruction, having to choose one or the other. Modern linguistic practices, such as translanguaging, allow us to overcome this subtractive way of interpreting the multilingual learning, to aspire to the legitimation of interculturality and multilingualism of the individuals, depriving these two concepts of any stereotype and ethnic prejudice. In the article we pointed out the possibilities of a successful application of the linguistic practice of translanguaging in the bilingual Italian-Slovenian context and the possibility to use it to create a positive intercultural learning enviroment.

Key words: multilingualism, interculture, translanguaging, bilingualism, language teaching 